\title{
Terminal augmented ureteroplasty with bladder onlay flap technique for recurrent distal ureteral stricture after ureteroneocystostomy: an initial case series
}

\author{
Yucai Wu ${ }^{1,2,3 \#}$, Weijie Zhu ${ }^{1,2,3 \#}$, Kunlin Yang ${ }^{1,2,3 \#}$, Shubo Fan ${ }^{1,2,3}$, Bao Guan ${ }^{1,2,3}$, Bingwei Huang ${ }^{4}$, \\ Jie Wang ${ }^{1,2,3}$, Jianxin Wang ${ }^{1,2,3}$, Zhihua $\mathrm{Li}^{1,2,3}$, Hua Guan ${ }^{1,2,3}$, Yanbo Huang ${ }^{1,2,3},{\text { Zhe } \text { Li }^{4} \text {, Peng Zhang }}^{4}$, \\ Xuesong $\mathrm{Li}^{1,2,3}$, Liqun Zhou ${ }^{1,2,3}$ \\ ${ }^{1}$ Department of Urology, Peking University First Hospital, Beijing, China; ${ }^{2}$ Institute of Urology, Peking University; National Urological Cancer \\ Centre, Beijing, China; ${ }^{3}$ Urogenital Diseases (Male) Molecular Diagnosis and Treatment Center, Peking University; Beijing, China; ${ }^{4}$ Department of \\ Urology, Emergency General Hospital, Beijing, China \\ Contributions: (I) Conception and design: Y Wu, W Zhu, X Li; (II) Administrative support: L Zhou, P Zhang; (III) Provision of study materials or \\ patients: W Zhu, K Yang, S Fan; (IV) Collection and assembly of data: B Guan, B Huang, J Wang; (V) Data analysis and interpretation: Z Li, H \\ Guan, Y Huang; (VI) Manuscript writing: All authors; (VII) Final approval of manuscript: All authors. \\ "These authors contributed equally to this work. \\ Correspondence to: Peng Zhang. Department of Urology, Emergency General Hospital, 29 Xiba Henan Li, Chaoyang, Beijing 100028, China. \\ Email: punky_bird@hotmail.com; Xuesong Li. Department of Urology, Peking University First Hospital, 8 Xishiku Street, Xicheng, Beijing 100034, \\ China. Email: pineneedle@sina.com.
}

Background: Bladder flap has been shown to be a feasible treatment for distal ureteral stenosis; this technique has been improved such that it can be used to address complex urinary tract obstructions. The purpose of the present study was to describe a surgical technique of ureteroplasty with a bladder onlay flap, which consists of a nontransecting and terminal augmented anastomosis, for repairing recurrent distal strictures of the ureter.

Methods: We retrospectively reviewed 6 patients who underwent this procedure between May 2018 and November 2019. These patients were diagnosed with distal ureteral stenosis and had previously undergone ureteroneocystostomy (one with a Boari flap) but suffered recurrence of flank pain. Patient characteristics, perioperative data and follow-up outcomes were gathered. The success of the operation was judged by symptomatic relief (subjective success) and improved radiographic imaging and renal function (objective success).

Results: Preoperative computed tomography urography (CTU) showed hydronephrosis in all patients: severe hydronephrosis was observed in $83.3 \%$ of patients $(5 / 6)$, and moderate hydronephrosis was observed in $16.7 \%(1 / 6)$. The mean stricture length was $2 \mathrm{~cm}$. The mean operating time, estimated blood loss and postoperative hospital stays of the six patients were $193.3 \mathrm{~min}(160-270 \mathrm{~min}), 41.5 \mathrm{~mL}(10-58 \mathrm{~mL})$ and 8.2 days (6-11 days), respectively. No serious complications (Clavien-Dindo grade $\geq 3$ ) occurred during or after the operations. The mean follow-up time was 24.5 months (range, 14 to 29). The objective success rate was $83.3 \%(5 / 6)$, and the subjective success rate was $100 \%$.

Conclusions: Our technique of ureteroplasty with a bladder onlay flap by nontransecting and terminal augmented anastomosis is feasible and improves the recovery rate after the repair of recurrent distal ureteral stenosis. Patients who have had previous unsuccessful surgeries might benefit from this approach.

Keywords: Bladder onlay flap; recurrent distal ureteral stenosis; ureteroneocystostomy

\footnotetext{
$\wedge$ ORCID: 0000-0003-2699-8377.
} 
Submitted Mar 22, 2021. Accepted for publication Jul 08, 2021.

doi: $10.21037 /$ tau-21-252

View this article at: https://dx.doi.org/10.21037/tau-21-252

\section{Introduction}

Ureter stricture disease (USD) may result from various causes, such as malignancy, stones, fibrosis, radiotherapy, infection and trauma (1). Although continuity of the lumen is not interrupted, USD may cause varying degrees of upper urinary tract obstruction and hydronephrosis. The choice of treatment for USD depends on the location and length of stenosis, and the reconstructive surgery remains a challenge for urologists (2).

One treatment for distal USD is ureteroneocystostomy with or without a Boari flap. These methods are used to repair ureteral defects of 4-5 and 6-10 $\mathrm{cm}$ respectively (3). In addition, other urinary tract reconstructive approaches, including ureteroureterostomy, ureteroneocystostomy with or without a psoas hitch and ureteral reimplantation with or without a psoas hitch, have been proven to be effective in repairing distal USD $(4,5)$. However, tight adhesion and periureteral fibrosis greatly reduce the success rate of these treatments. In addition, the required transection of the ureter in these techniques may impair vascularity and contribute to recurrent strictures. Postoperative scarring and fibrosis often result in ischemic tissue injury. In this condition, scar tissue needs to be removed and replaced by healthy and vascularized tissue for reanastomosis during a second urinary tract reconstruction. Few publications have examined minimally invasive techniques to manage USD associated with ischemic injury (6). Most of the evidence is based on single-center and retrospective studies without long-term follow-up (7-9).

In this article, we present our laparoscopic bladder onlay flap procedure with a nontransecting and terminal augmented anastomosis for patients who had previously undergone treatment with ureteroneocystostomy (one with a bladder flap) but suffered a recurrence of flank pain. To our knowledge, this is the first report on this novel technique. We present the following article in accordance with the AME Case Series checklist (available at https:// dx.doi.org/10.21037/tau-21-252).

\section{Methods}

\section{Study population}

Between May 2018 and November 2019, 6 patients who had recurrent distal USD after ureteroneocystostomy were treated by terminal augmented ureteroplasty with the bladder onlay flap technique. The preoperative 3 -dimensional reconstruction models of computed tomography urography (CTU) suggested that each patient had distal USD and indicated severe hydronephrosis in 5 patients and moderate hydronephrosis in 1 patient (Figure $1 A$ ).

All procedures performed in this study were in accordance with the Declaration of Helsinki (as revised in 2013) and approved by the Ethics Committee of Peking University First Hospital (approval number: 2019-134). Because of the retrospective nature of the research, the requirement for informed consent was waived.

\section{Surgical technique}

Patients were positioned in the modified flank position with the disease side elevated by $30^{\circ}$, and a 3-cavity Foley catheter was placed into the bladder before surgery. The following trocars were placed after establishing pneumoperitoneum (taking the right affected side as an example): $10 \mathrm{~mm}$ below the umbilicus, $10 \mathrm{~mm}$ in the lateral margin of rectus abdominis muscle at umbilical level, and $5 \mathrm{~mm}$ at the midpoint between the umbilicus and pubic symphysis (Figure 1B).

The surgery was begun by identifying the affected ureter. After the colon adhesion was separated and the retroperitoneum was opened, the ureter was found along the upper part of the iliac blood vessels. The stenosis position was confirmed near the ureterovesical junction when the ureter was dissociated from the pelvic segment to the end. The bladder was suspended, and a longitudinal ureterotomy was made proximal to the ureteral stenosis 

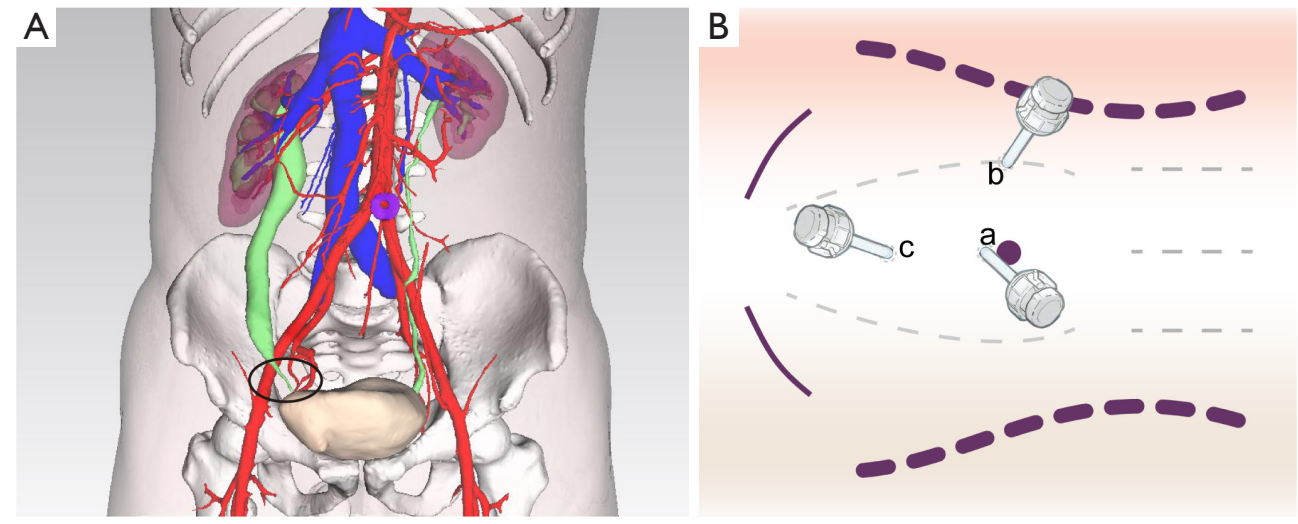

Figure 1 Three-dimensional reconstruction models of computed tomography urography (CTU) and surgical trocar placement. (A) Threedimensional reconstruction of the preoperative CTU (Patient 5). (B) Surgical trocar placement: (a) below the umbilicus. (b) the lateral margin of the rectus abdominis muscle at the umbilical level. (c) the midpoint between umbilicus and symphysis pubis.
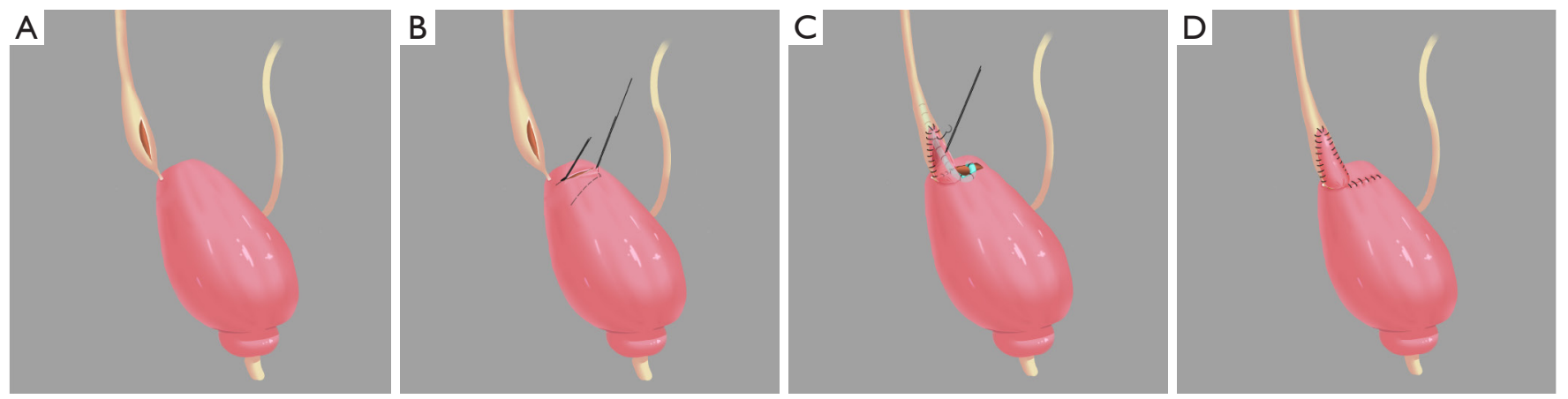

Figure 2 Schematic illustration of surgical procedures. (A) The longitudinal ureterotomy was made proximal to the ureteral stricture. (B) The trapezoidal bladder flap was taken along the extension of the distal strictured ureter. (C) The bladder flap was anastomosed side to side with the lateral wall of the incised ureter. (D) The free edges of the bladder wall were sutured.

(Figure 2A). Then, the length of the stricture was measured, and a trapezoidal bladder onlay flap was taken along the extension of the distal ureter (Figure $2 B$ ). Next, the distal segment of the ureter stricture was selected as the base, and the trapezoidal onlay flap was turned to the side to ensure complete coverage of the stricture. Anastomosis was performed using a running 4-0 absorbable suture. The tip of the onlay flap was fixed proximal to the strictured ureter, and one lateral wall of the bladder onlay flap was anastomosed side to side with the lateral wall of the incised ureter (Figure 2C). Then, a double J stent was placed into the ureter and bladder crossing the anastomosis. When completed, the remaining bladder onlay flap wall was anastomosed with the medial wall of the incised ureter in a side-to-side way. The free edges of the bladder wall were sutured (Figure 2D). No anastomotic leakage of urine was found after injecting normal saline into the bladder. A drainage tube was placed near the anastomosis, and the previously placed Foley catheter remained in the bladder. The actual intraoperative diagram is shown in Figure 3 and Video 1.

\section{Postoperative management and follow-up}

The drainage tube was pulled out when the daily drainage volume was less than $50 \mathrm{~mL}$, and the drainage creatinine level was comparable to the serum creatinine level. The Foley catheter was removed after two weeks if there was no leak on the cystogram. The double $\mathrm{J}$ stent was removed after 2-3 months. During follow-up, patients were advised to obtain a renal ultrasound every month for the first two years and then every three months thereafter. CTU and 

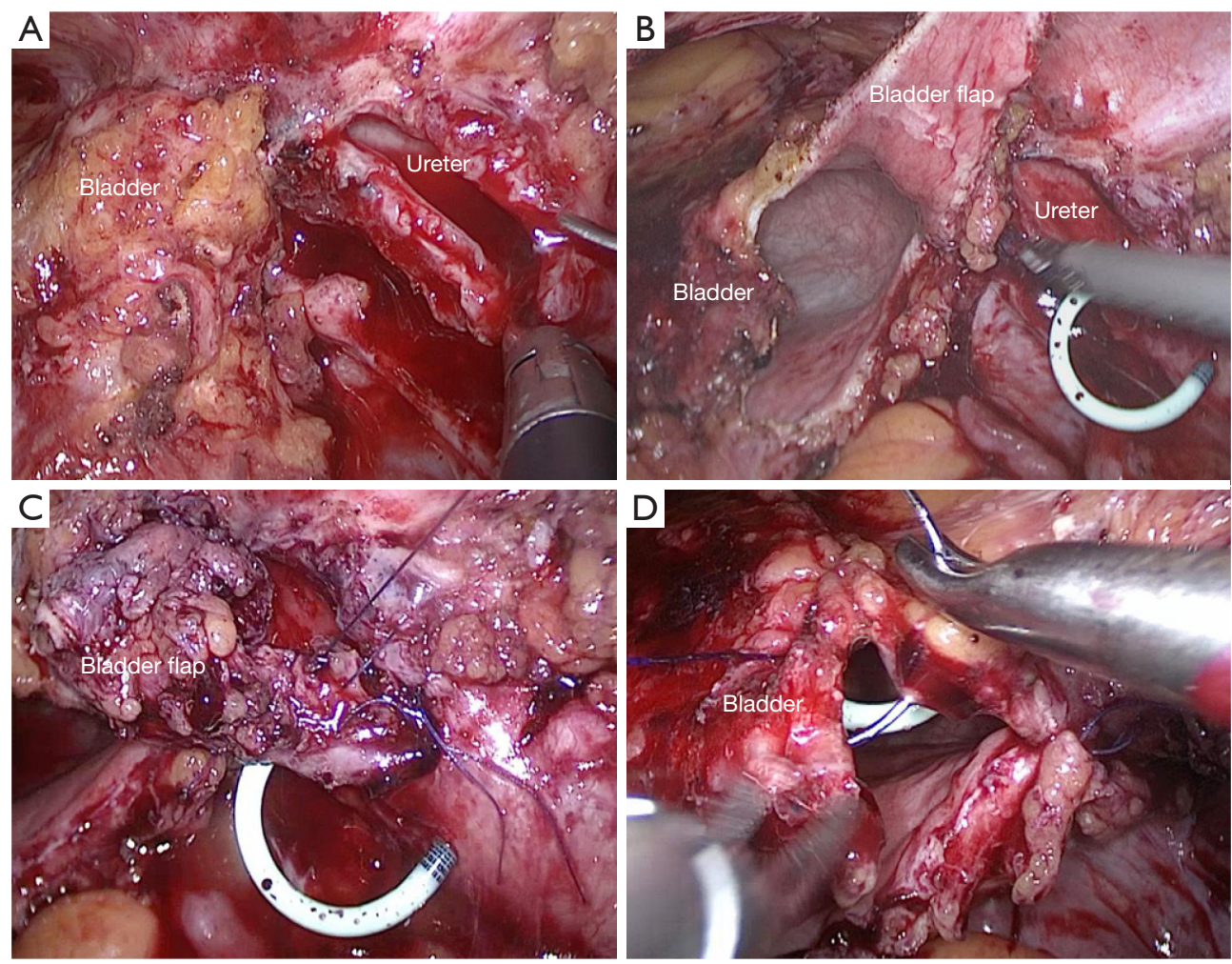

Figure 3 Surgical procedures of terminal augmented ureteroplasty with the bladder onlay flap technique. (A) The stenosis position was confirmed near the ureterovesical junction and a longitudinal ureterotomy was made proximal to the ureteral stricture. (B) The bladder flap was made along the extension of the distal strictured ureter. (C) The bladder flap was anastomosed with the lateral wall of the incised ureter in a side-to-side way. (D) The free edges of the bladder wall were sutured.

diuretic nuclear renography were recommended at 3 and 6 months after removing the double J stent. The evaluation index for a successful operation is divided into a subjective index and an objective index. The subjective index referred to relief of symptoms, and the objective index was defined as decreased hydronephrosis on CTU with three-dimensional reconstruction models and improved differential renal function on nuclear renography.

\section{Statistical analysis}

Excel software (version 2019) was used for data management. All analyses were performed with SPSS version 22.0 software (SPSS, Inc., IBM Corp., Somers, NY, USA). The quantitative variables included age, stricture length, operative time, estimated blood loss, postoperative hospital stays, duration of catheterization, duration of stenting and follow-up time. The qualitative variables included sex, affected side and surgical complications.

\section{Results}

Demographic and preoperative characteristics of patients are shown in Table 1. The group consisted of 5 females and 1 male. The age ranged from 16 to 63 years old, with a mean age of 38.8 years old. Etiology for the primary stricture in our cohort included congenital causes (4/6), iatrogenic injury (1/6) and inflammation (1/6). The patients previously underwent ureteroneocystostomy (one combined with a Boari flap). They were found to have recurrent hydronephrosis due to flank pain. The mean preoperative serum creatinine was $0.9 \mathrm{mg} / \mathrm{dL}(0.6-1.5 \mathrm{mg} / \mathrm{dL})$. The mean stricture length was $2.0 \mathrm{~cm}(1.5-3.0 \mathrm{~cm})$.

Perioperative and outcome data are presented in Table 2. The mean operative time was $193.3 \mathrm{~min}$ (160-270 $\mathrm{min}$ ), and the mean blood loss was $41.5 \mathrm{~mL}(10-58 \mathrm{~mL})$. The mean postoperative hospital stay was 8.2 days (6-11 days).

The Foley catheter and double-J stent were removed on average 14.67 days and 80 days after surgery, respectively.

No intraoperative complications or postoperative 
Table 1 Patient demographics and preoperative data of patients

\begin{tabular}{|c|c|c|c|c|c|c|c|c|c|}
\hline Case & Age & Gender & $\begin{array}{l}\text { Affected } \\
\text { side }\end{array}$ & $\begin{array}{l}\text { Prior surgeries } \\
\text { for stricture }\end{array}$ & $\begin{array}{l}\text { Presenting } \\
\text { symptoms }\end{array}$ & $\begin{array}{c}\text { Stricture } \\
\text { length }(\mathrm{cm})\end{array}$ & Preoperative imaging & $\begin{array}{l}\text { GFR of affected } \\
\text { side (mL/min) }\end{array}$ & $\begin{array}{c}\mathrm{SCr} \\
(\mathrm{mg} / \mathrm{dL})\end{array}$ \\
\hline Patient 1 & 33 & $\mathrm{~F}$ & Right & 1 & Flank pain & 1.5 & Severe hydronephrosis & 37.84 & 0.9 \\
\hline Patient 2 & 30 & $\mathrm{~F}$ & Right & 3 & Flank pain & 1.5 & Severe hydronephrosis & $N G$ & 0.6 \\
\hline Patient 3 & 32 & $\mathrm{~F}$ & Right & 1 & Flank pain & 2 & Severe hydronephrosis & 60 & 0.6 \\
\hline Patient 6 & 16 & M & Right & 1 & Flank pain & 3 & Severe hydronephrosis & $N G$ & 1 \\
\hline
\end{tabular}

GFR, glomerular filtration rate; NG, no given; SCr, serum creatinine.

complications (Clavien-Dindo grade $\geq 3$ ) were reported (10). Serum creatinine returned to normal values after surgery. All patients had clinical success, defined as relief of flank pain; two patients still had mild flank pain, but it was a significant improvement compared to their preoperative pain. In addition, $83.3 \%$ of patients were found to have objective success in which CTU showed improvement of hydronephrosis during a mean follow-up period of 24.5 months (14-29 months).

\section{Discussion}

The incidence of USD has increased dramatically due to the introduction and widespread use of endoscopy. Latrogenic injury occurring in urologic, gynecologic, and general surgical procedures is a major cause of USD. Most of the distal ureteral stenosis caused by injury related to gynecological surgery is due to the embryologic relationship and anatomic proximity between the reproductive organs and the ureters (11). It has been reported that the incidence of ureteral injury during pelvic surgery is $1 \%$ to $10 \%$ (12). Other causes of distal USD include congenital anomalies, inflammation, tumors and ischemia. Endoscopic, open, laparoscopic and robot-assisted techniques are available to treat USD, giving surgeons different choices according to the location and length of USD (2). Compared with open surgery, these minimally invasive techniques have the advantages of less intraoperative blood loss and postoperative pain, faster recovery and shorter hospital stays. However, endoscopic treatment has a poor success rate in patients with stenosis longer than $2 \mathrm{~cm}$, stenosis associated with radiotherapy or ischemic injury and stenosis at a mid-ureteral position (6). For distal USD, because of the narrow space and complex anatomy of the pelvis, it is difficult for surgeons to protect an adequate blood supply to the ureter, which could decrease the risk of recurrent stricture after surgery.

Ureteroneocystostomy with or without a psoas hitch or Boari flap is considered the most appropriate way to manage distal USD, and it is assumed that Boari flap ureteroneocystostomy is useful in higher and more extensive ureteric defects than bladder psoas-hitch fixation (13). The bladder flap is an ideal treatment of choice due to its abundant blood supply. In addition, the bladder flap has great histocompatibility with the ureter to minimize the occurrence of anastomotic stenosis, urinary fistula and other complications (14). This technique is mostly used to bridge defects of the distal ureter and is reliable in reconstruction of ureteral proximal strictures by renal mobilization with downward nephropexy (15). It has been reported that a modified Boari flap could be utilized to reconstruct the ureter in complex oncological cases (16). Currently, robotic surgery is widely used in clinical practice, and robotic Boari flaps have been successfully performed to manage distal USD (17).

In our retrospective case series, all six patients had unsuccessful treatment previously with ureteroneocystostomy with or without a Boari flap, which made it difficult to mend the stricture by ureteroneocystostomy. Moreover, previous operations have caused scar and severe abdominal adhesions, leading to an increased degree of surgical difficulty. In recent years, ureteral reimplantation via a nontransecting side-to-side anastomosis was verified as an effective operation for distal USD but was limited after multiple reconstructive surgeries (18). In our study, we applied terminal augmented ureteroplasty with the bladder onlay flap technique, which allowed reparation of longer ureteral stenosis and tension-free anastomosis. The bladder 


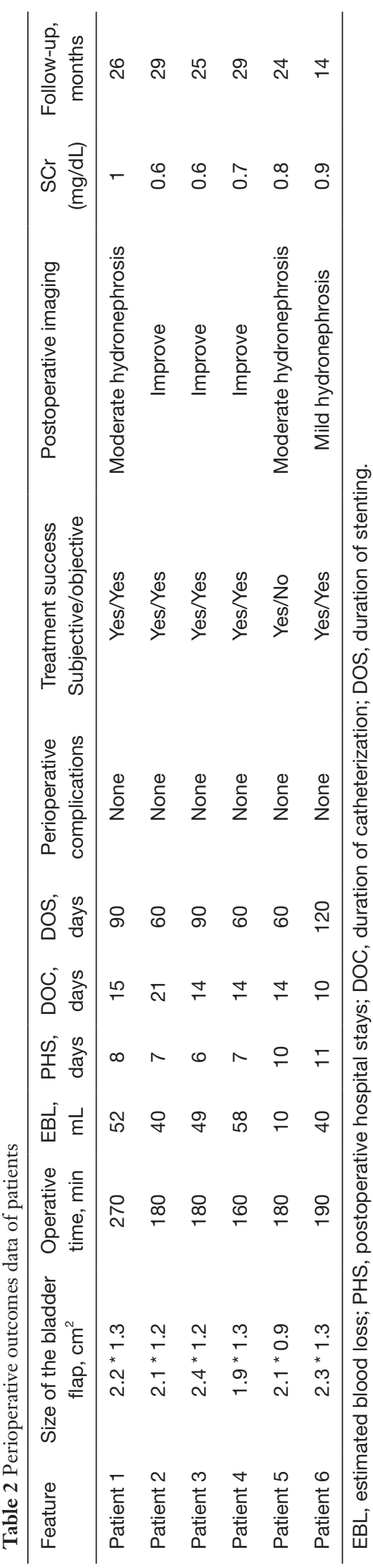

onlay flap required for this operation was smaller than the traditional Boari flap, and augmentation of the terminal ureter was suitable for nonobliterated ureteral strictures.

Based on our experience, there are technical considerations about terminal augmented ureteroplasty with the bladder onlay flap technique. First, one of the difficulties in distal USD reconstructive surgery after previous repair is to identify the position of the strictured ureter. The position of the iliac vessels and ureter should be carefully identified during the operation due to extensive fibrosis around the ureter and the loss of normal tissue anatomy. The surgeon should avoid mistaking the obturator vessel, gonadal vessel and superior vesicle vessel for the ureter when searching for a strictured ureter. However, it contributes to finding the ureter when the proximal ureter is obviously dilated in some patients. Second, the key of this reconstructive operation is to protect the ureter so that it has an adequate blood supply and tension-free anastomosis. Care should be taken to protect the peripheral tissue surrounding the ureter and its inherent ureteral blood supply (19). To avoid excessive tension at the anastomosis, the bladder and pelvic wall tissues around the anastomosis were fixed on the lumbar tendon arch above iliac vessels. Third, the bladder onlay flap was taken adjacent to the distal strictured ureter and performed with a terminal augmented anastomosis rather than end-to-end or end-to-side anastomosis, which expanded the lumen of the terminal ureter and relieved the tension of the anastomosis. Moreover, our operation is not required to transect the distal strictured ureter, reducing the risk of destroying ureteral blood transport. Therefore, the anastomotic site has a good blood supply and low tension, which effectively avoids complications such as urinary fistula and anastomotic stenosis.

The limitations of our research are its small sample size and retrospective nature. In addition, the followup period was too short to draw a definitive long-term conclusion. What is gratifying is that all six patients' flank pain improved after surgery. CTU suggested that hydronephrosis was relieved in 5 patients $(83.3 \%)$, and none developed evidence of ureteral obstruction.

\section{Conclusions}

In conclusion, our experience with terminal augmented ureteroplasty with the bladder onlay flap technique is recommended to repair complex distal USDs even for patients who had previously failed ureteroneocystostomies. Prospective studies with longer follow-up durations and 
larger patient cohorts are needed to assess long-term outcomes of the technique.

\section{Acknowledgments}

The authors thank the entire staff of the Department of Urology, Peking University First Hospital.

Funding: None.

\section{Footnote}

Reporting Checklist: The authors have completed the AME Case Series checklist. Available at https://dx.doi. org/10.21037/tau-21-252

Data Sharing Statement: Available at https://dx.doi. org/10.21037/tau-21-252

Peer Review File: Available at https://dx.doi.org/10.21037/ tau-21-252

Conflicts of Interest: All authors have completed the ICMJE uniform disclosure form (available at https://dx.doi. org/10.21037/tau-21-252). Dr. XL serves as an unpaid editorial board member of Translational Andrology and Urology. The other authors have no conflicts of interest to declare.

Ethical Statement: The authors are accountable for all aspects of the work in ensuring that questions related to the accuracy or integrity of any part of the work are appropriately investigated and resolved. All procedures performed in this study were in accordance with the Declaration of Helsinki (as revised in 2013) and approved by the Ethics Committee of Peking University First Hospital (approval number: 2019-134). Because of the retrospective nature of the research, the requirement for informed consent was waived.

Open Access Statement: This is an Open Access article distributed in accordance with the Creative Commons Attribution-NonCommercial-NoDerivs 4.0 International License (CC BY-NC-ND 4.0), which permits the noncommercial replication and distribution of the article with the strict proviso that no changes or edits are made and the original work is properly cited (including links to both the formal publication through the relevant DOI and the license). See: https://creativecommons.org/licenses/by-nc-nd/4.0/.

\section{References}

1. Tyritzis SI, Wiklund NP. Ureteral strictures revisited... trying to see the light at the end of the tunnel: a comprehensive review. J Endourol 2015;29:124-36.

2. Png JC, Chapple CR. Principles of ureteric reconstruction. Curr Opin Urol 2000;10:207-12.

3. Nakada SY, Best SL. Management of Upper Urinary Tract Obstruction. PA: Elsevier, 2016.

4. Ghosh B, Jain P, Pal DK. Managing Mid and Lower Ureteral Benign Strictures: The Laparoscopic Way. J Laparoendosc Adv Surg Tech A 2018;28:25-32.

5. Wenske S, Olsson CA, Benson MC. Outcomes of distal ureteral reconstruction through reimplantation with psoas hitch, Boari flap, or ureteroneocystostomy for benign or malignant ureteral obstruction or injury. Urology 2013;82:231-6.

6. Hafez KS, Wolf JS Jr. Update on minimally invasive management of ureteral strictures. J Endourol 2003; 17:453-64.

7. Richter F, Irwin RJ, Watson RA, et al. Endourologic management of benign ureteral strictures with and without compromised vascular supply. Urology 2000;55:652-7.

8. Reus C, Brehmer M. Minimally invasive management of ureteral strictures: a 5-year retrospective study. World J Urol 2019;37:1733-8.

9. Bourdoumis A, Kachrilas S, Kapoor S, et al. The use of a thermoexpandable metal alloy stent in the minimally invasive management of retroperitoneal fibrosis: a single center experience from the United kingdom. J Endourol 2014;28:96-9.

10. Mitropoulos D, Artibani W, Biyani CS, et al. Validation of the Clavien-Dindo Grading System in Urology by the European Association of Urology Guidelines Ad Hoc Panel. Eur Urol Focus 2018;4:608-13.

11. Gilmour DT, Das S, Flowerdew G. Rates of urinary tract injury from gynecologic surgery and the role of intraoperative cystoscopy. Obstet Gynecol 2006;107:1366-72.

12. Pastore AL, Palleschi G, Silvestri L, et al. Endoscopic rendezvous procedure for ureteral iatrogenic detachment: report of a case series with long-term outcomes. J Endourol 2015;29:415-20.

13. Andrade C, Narducci F, Bresson L, et al. Boari flap ureteroneocystostomy in an oncological patient. Gynecol Oncol 2016;143:193.

14. Aminsharifi A. Minimally Invasive Management of 
Concomitant Vesicovaginal and Ureterovaginal Fistulas After Transabdominal Hysterectomy: Laparoscopic Vesicovaginal Fistula Repair With Ureteroneocystostomy Using a Boari Flap. J Minim Invasive Gynecol 2018;25:17-8.

15. Mauck RJ, Hudak SJ, Terlecki RP, et al. Central role of Boari bladder flap and downward nephropexy in upper ureteral reconstruction. J Urol 2011;186:1345-9.

16. Radtke JP, Korzeniewski N, Huber J, et al. Ureterocystoneostomy in complex oncological cases with an "Uebelhoer" modified Boari bladder flap. Langenbecks

Cite this article as: $\mathrm{Wu} \mathrm{Y,} \mathrm{Zhu} \mathrm{W,} \mathrm{Yang} \mathrm{K,} \mathrm{Fan} \mathrm{S,} \mathrm{Guan} \mathrm{B,}$ Huang B, Wang J, Wang J, Li Z, Guan H, Huang Y, Li Z, Zhang P, Li X, Zhou L. Terminal augmented ureteroplasty with bladder onlay flap technique for recurrent distal ureteral stricture after ureteroneocystostomy: an initial case series. Transl Androl Urol 2021;10(8):3332-3339. doi: 10.21037/tau21-252
Arch Surg 2017;402:1271-8.

17. Sagalovich D, Garisto J, Bertolo R, et al. Minimally Invasive Management of Ureteral Distal Strictures: Robotic Ureteroneocystostomy With a Bilateral Boari Flap. Urology 2018;120:268.

18. Slawin J, Patel NH, Lee Z, et al. Ureteral Reimplantation via Robotic Nontransecting Side-to-Side Anastomosis for Distal Ureteral Stricture. J Endourol 2020;34:836-9.

19. Stein R, Rubenwolf P, Ziesel C, et al. Psoas hitch and Boari flap ureteroneocystostomy. BJU Int 2013;112:137-55. 